\title{
вмј Global Health Why we are still failing to measure the nutrition transition
}

\author{
Helen L Walls, ${ }^{1}$ Deborah Johnston, ${ }^{2}$ Jacob Mazalale, ${ }^{3}$ Ephraim W Chirwa ${ }^{3}$
}

To cite: Walls HL, Johnston D, Mazalale J, et al. Why we are still failing to measure the nutrition transition. BMJ Glob Health 2018;3:e000657. doi:10.1136/ bmjgh-2017-000657

Received 23 November 2017 Revised 4 January 2018 Accepted 30 January 2018
Check for updates

${ }^{1}$ Department of Global Health and Development, Faculty of Public Health and Policy, London School of Hygiene and Tropical Medicine, London, UK ${ }^{2}$ Department of Economics, SOAS University of London, London, UK

${ }^{3}$ Department of Economics, University of Malawi Chancellor College, Zomba, Malawi

Correspondence to

Dr Helen L Walls;

helen.walls@Ishtm.ac.uk

\section{INTRODUCTION}

Malnutrition in all its forms-both underweight and micronutrient deficiencies, as well as overweight, obesity and associated non-communicable disease-is a global health issue, with the majority of cases arising in low-income and middle-income countries (LMICs). ${ }^{1}$ Much of this malnutrition, particularly shifts towards an increased prevalence of overweight and obesity, is driven by dietary changes described as the "nutrition transition', whereby populations move from traditional diets high in fibre and micronutrients, to more highly processed diets high in sugar, fat, salt, low in fibre and less nutrient densewith these dietary changes accompanied by changes in eating behaviours and physical activity patterns. $^{2}$

Ultraprocessed food products have been defined by Monteiro et al as "not entirely or mostly made from foods, but from industrial ingredients and additives, and are highly profitable'. ${ }^{3}$ Distinctions are made between processed foods that have undergone limited processing and are not so problematic for diets (or are indeed healthy) and more highly processed or 'ultraprocessed' products that are often particularly unhealthy (eg, burgers, frozen pasta, pizza and pasta dishes, nuggets and sticks, crisps, biscuits, bakery goods, confectionary, cereal bars, sugary drinks). ${ }^{3}$

As is widely recognised in public health, quality data are essential for the development of appropriate health policy and programmes. Thus, surveillance of trends in dietary patterns based on appropriate measurement is critical for understanding the nutrition transition in different country and community contexts and to inform an appropriate response. But despite the high burden of malnutrition, relatively little is known about actual food consumption in many populations and factors affecting this. Furthermore, there is recognised need for development of improved methods and metrics for agri-health research. ${ }^{3}{ }^{4}$ As we describe, standardised

\section{Summary box}

Much of the global burden of malnutrition, particularly shifts towards an increased prevalence of overweight and obesity, is driven by dietary changes described as the 'nutrition transition'.

- The nutrition transition appears to be driven by shifts in diets towards more 'ultraprocessed' foods high in sugar, fat, salt, low in fibre and less nutrient dense - but little is known about actual food consumption in many populations.

- Standard instruments used for measuring diets in low-income and middle-income countries are inadequate for assessing changing diets in the context of the nutrition transition, as they do not provide appropriate provision in response to categories for consumption of ultraprocessed food products.

- There is a need for a validated standardised food frequency questionnaire designed to measure changing dietary patterns that have appropriate provision for highly processed food types - and with the implementation of such questonnaires there is a need to consider food source and the importance of capturing foods eaten outside of the home as well as in the home.

dietary assessment instruments are in their current form inadequate for assessing diets in the context of the nutrition transition.

\section{Studies of the nutrition transition}

While a considerable body of literature assesses the nutrition transition in various country contexts, most such studies do not evaluate changes in dietary consumption of ultraprocessed food products. Instead, they measure changes in proxies of nutritional change-changes in anthropometric measures such as underweight, overweight and obesity or changes in food environments. Although studies of proxies of nutritional change constitute an important contribution to the literature, as Turner et $a l^{4}$ describe, data on actual consumption is necessary for a fuller understanding of the drivers of dietary change and policy 
responses ${ }^{4}$ —at least in the absence of gold-standard dietary intake surveys that are usually too expensive for widespread use in LMICs.

Studies examining the nutrition transition frequently measure changes in anthropometry, such as body weight and associated measures and/or health outcomes (cf. Morshed $e t a l$ and Lee $e t a l^{56}$ ), and characteristics of food environments such as food availability (cf. Morshed et a $a \tilde{P})$. Where changes in diets have been assessed, these have often been measured using broad food categories (eg, bread; cereals, porridge or other grains; root and tubers; beans and other legumes; eggs; milk; fermented milk or yoghurt; cheese and other dairy products; poultry; other meat; fish and shellfish; fruits; vegetables; oils, butter and other fats) (cf. Morshed $e t a l$ and Lee $e t a \tilde{l}^{6}$ ).

A small subset of studies of the nutrition transition have, however, explicitly measured consumption of ultraprocessed food products (eg, deep fried foods, soft drinks, snack foods, instant foods, processed meats and bakery goods) in their dietary surveys (cf. Morshed et al and Kelly et $a \tilde{l}^{7}$ ). A few studies have described the development of food frequency questionnaires to address the nutrition transition in particular contexts (South Africa, South India, Sri Lanka) (cf. Shaikh et al, Zingoni et al, Jayawardena $\left.e t a l^{8-10}\right)$.

\section{The lack of appropriate instruments for measuring the nutrition transition}

The quality of studies assessing the nutrition transition to a large part reflects the quality of the standard instruments available for assessing diets. The standard instruments for assessing dietary diversity and food security in LMICs-which include dietary diversity surveys, household expenditure surveys and household food security surveys-are in their current form largely inadequate for assessing diets in the context of the nutrition transition.

Dietary diversity is commonly measured by the Household Dietary Diversity Score (HDDS), which assesses household access to a variety of foods, and at individual level, by the Minimum Dietary Diversity for Women of Reproductive Age tool (MDD-W) ${ }^{11}$ and WHO Infant and Young Child Minimum Dietary Diversity Tool (IYCF-MDD $),{ }^{12}$ both indicators of individual nutrient adequacy of the diet. However, these instruments ask about broad food categories but do not address ultraprocessed food types. While some ultraprocessed foods would be relatively simple to include in these questionnaires (ie, in the HDDS, bakery goods or biscuits under the category asking about high carbohydrate food products), they would often be included in the same broad category as more healthy foods such as rice, oats or quinoa. Additionally, many highly processed foods such as a burger that do not obviously fit a category may be split into multiple categories-between, for example, categories addressing 'breads', 'meat' and 'vegetables'-and potentially appear as a relatively healthy dietary pattern. While the MDD-W and IYCF-MDD do include 'sweets' and 'sugar sweetened beverages' as separate categories, this is insufficient to address the range of foods contributing to the nutrition transition.

Household food security is often measured using the Food Insecurity Experience Scale. ${ }^{13} 14$ However, this measure addresses a lack of access to foods, for example, missing meals due to being unable to afford food. Thus, it is a measure of food security rather than of dietary diversity.

\section{Need for standardised dietary assessment instruments that address the nutrition transition}

The standardised instruments for measuring diets are in their current form inadequate for measuring the nutrition transition given they do not provide appropriate provision in response categories for consumption of ultraprocessed food products. While such assessment tools need to be able to be used easily, rapidly and at low cost, they also need to be fit for purpose. In the context of the nutrition transition, they are not fit for purposeproblematic, given that quality data are essential to inform appropriate health policy and programme development and implementation. The variables included and not included in a dataset are of great significance given that what is not measured is far less likely to be addressed.

While some instruments capturing ultraprocessed food consumption have been developed for particular country contexts, ${ }^{8-10}$ there is no standardised dietary assessment tool for measuring such food consumption and associated dietary change. Thus, despite the importance of understanding better changing dietary patterns, very little is actually known about what people eat and how this is changing over time.

There is a need for a validated standardised food frequency questionnaire that is designed to be able to measure changing dietary patterns that includes ultraprocessed food types. While there will always be the need for such standardised questionnaires to be tailored to local contexts, a standardised model questionnaire enabling the identification of trends in the consumption of ultraprocessed foods would provide the model for basing more contextualised dietary assessment.

A key benefit of dietary assessment instruments such as food frequency questionnaires is their rapid, userfriendly, easily administered and low-cost nature, as opposed to gold-standard dietary intake surveys. ${ }^{11}$ But with changing consumption patterns, it will be important for such surveys not only to include questions that capture ultraprocessed foods. Also important is the consideration of food source. Such surveys need to include not only foods eaten in the home but also foods eaten outside of the home-which given their nature are more likely to be ultraprocessed. In many LMICs, 'street foods' have shifted from being traditional dishes to more typically western-style fast foods, and as reviewed by Steyn et $a l^{15}$, this shift has significantly altered levels of macronutrient and micronutrient consumption. Of importance, here is the distinction between household and individual-level food frequency questionnaires, as the former 
in its standardised form excludes foods eaten outside of the home-problematic in the context of measuring ultraprocessed food consumption. Thus, not only do standardised dietary assessment tools need appropriate provision for the inclusion of ultraprocessed foods, but individual-level assessments that include foods eaten outside the home are also critical if the consumption of such foods is to be adequately measured.

\section{CONCLUSION}

Given the considerable global burden of disease posed by malnutrition and changing dietary patterns as a key driver of this, understanding these changing dietary patterns forms a crucial part of informing an appropriate policy response. However, the standardised dietary assessment tools are not in their current form suitable for measuring these crucial changes. The standardised instruments currently commonly used to assess diets in LMICs are not appropriate for measuring shifts to ultraprocessed foods. There is need for standardised instruments assessing dietary diversity that are appropriate for measuring changes towards increased consumption of ultraprocessed foods that would provide the model for more contextualised, locally appropriate dietary assessment. Given that such foods are often consumed outside the home, individual dietary assessment that includes foods eaten outside the home as well as in the homerather than household-level dietary assessment that only includes the latter-will also be critical.

Acknowledgements This research has been funded by the Drivers of Food Choice (DFC) Competitive Grants Programs, which is funded by the UK Government's Department for International Development and the Bill and Melinda Gates Foundation and managed by the University of South Carolina, Arnold School of Public Health, USA; however, the views expressed do not necessarily reflect the UK Government's official policies.

Contributors HW and DJ conceived the article and HW led the writing of the manuscript. All authors contributed to critical content and reviewed the final manuscript.

Funding This study was funded by Bill and Melinda Gates Foundation.

Competing interests None declared.

Provenance and peer review Not commissioned; externally peer reviewed.

Data sharing statement No additional data are available.
Open Access This is an Open Access article distributed in accordance with the Creative Commons Attribution Non Commercial (CC BY-NC 4.0) license, which permits others to distribute, remix, adapt, build upon this work non-commercially, and license their derivative works on different terms, provided the original work is properly cited and the use is non-commercial. See: http://creativecommons.org/ licenses/by-nc/4.0/

(c) Article author(s) (or their employer(s) unless otherwise stated in the text of the article) 2018. All rights reserved. No commercial use is permitted unless otherwise expressly granted.

\section{REFERENCES}

1. Bhutta ZA, Salam RA. Global nutrition epidemiology and trends. Ann Nutr Metab 2012;61:19-27.

2. Popkin BM. The nutrition transition in low-income countries: an emerging crisis. Nutr Rev 1994;52:285-98.

3. Monteiro CA, Moubarac JC, Cannon G, et al. Ultra-processed products are becoming dominant in the global food system. Obes Rev 2013;14:21-8.

4. Turner R, Hawkes C, Jeff W, et al. Agriculture for improved nutrition: the current research landscape. Food Nutr Bull 2013;34:369-77.

5. Morshed AB, Becker HV, Delnatus JR, et al. Early nutrition transition in Haiti: linking food purchasing and availability to overweight status in school-aged children. Public Health Nutr 2016;19:3378-85.

6. Lee MJ, Popkin BM, Kim S. The unique aspects of the nutrition transition in South Korea: the retention of healthful elements in their traditional diet. Public Health Nutr 2002;5:197-203.

7. Kelly M, Seubsman S-ang, Banwell C, et al. Thailand's food retail transition: supermarket and fresh market effects on diet quality and health. Br Food J 2014;116:1180-93.

8. Shaikh NI, Frediani JK, Ramakrishnan U, et al. Development and evaluation of a Nutrition Transition-FFQ for adolescents in South India. Public Health Nutr 2017;20:1162-72.

9. Zingoni C, Norris SA, Griffiths PL, et al. Studying a population undergoing nutrition transition: a practical case study of dietary assessment in urban South African adolescents. Ecol Food Nutr 2009;48:178-98.

10. Jayawardena R, Byrne NM, Soares MJ, et al. Validity of a food frequency questionnaire to assess nutritional intake among Sri Lankan adults. Springerplus 2016;5:162.

11. Kennedy G, Ballard T, Dop M. Guidelines for measuring household and individual dietary diversity. Rome: Nutrition and Consumer Protection Division, Food and Agriculture Organization of the United Nations, 2011.

12. World Health Organization. Indicators for assessing infant and young child feeding practices: part 2 measurement. Geneva: World Health Organization, 2010.

13. Food and Agriculture Organization of the United Nations (FAO). Measuring food insecurity through people's experiences 2016. www. fao.org/economic/ess/ess-fs/voices/en/

14. Food and Agriculture Organization of the United Nations (FAO). Global food insecurity experience scale survey modules: Food and Agriculture Organization, 2016.

15. Steyn NP, McHiza Z, Hill J, et al. Nutritional contribution of street foods to the diet of people in developing countries: a systematic review. Public Health Nutr 2014;17:1363-74. 